\title{
Fabry-Pérot-multichannel spectrometer tandem for ultra-high resolution Raman spectroscopy
}

\author{
Guillermo Rozas, ${ }^{1, a)}$ Bernard Jusserand, ${ }^{2}$ and Alejandro Fainstein ${ }^{1}$ \\ ${ }^{1}$ Centro Atómico Bariloche and Instituto Balseiro, CNEA, Bustillo 9500, R8402AGP S. C. de Bariloche, \\ Argentina \\ ${ }^{2}$ Institut des Nanosciences de Paris, Université Pierre et Marie Curie, CNRS UMR 7588, 4 Place Jussieu, \\ 75005 Paris, France
}

(Received 23 September 2013; accepted 20 December 2013; published online 15 January 2014)

\begin{abstract}
We present a novel ultra-high resolution Raman spectroscopy technique based in a Fabry-Pérot/triple spectrometer tandem with multichannel acquisition. We describe the system, detail the calibration process, and experimentally test the technique, showing that effective finesses in excess of 1000 are possible. The technique is specifically tailored for low intensity, complex and spectrally extended Raman spectra, providing shorter acquisition times with respect to similar tandem systems with monochannel detectors. (C) 2014 AIP Publishing LLC. [http://dx.doi.org/10.1063/1.4861345]
\end{abstract}

\section{INTRODUCTION}

In the last 30 years Raman spectroscopy has developed into a powerful tool to study acoustic vibrations in nanostructures, both for the characterization of the nanostructure and the phonons themselves. ${ }^{1-3}$ Experimental studies have been extensively presented in which the acoustic Raman spectrum in the $3-100 \mathrm{~cm}^{-1}$ range has provided information about the properties of the materials that form a nanostructure, and about the interaction between light, electrons, and sound in these systems. ${ }^{4-7}$

In most of the cases the resolution of a standard high resolution Raman spectrometer is enough to obtain the desired information. As a reference, a triple Raman spectrometer in additive configuration has an optimal resolution in the nearinfrared of $\sim 0.2 \mathrm{~cm}^{-1}(\sim 25 \mu \mathrm{eV})$. This number can be compared, for example, to the typical separation of around $2 \mathrm{~cm}^{-1}$ between the Raman peaks found in a semiconductor superlattice designed to study terahertz phonons. ${ }^{5}$ Unfortunately, in many interesting experiments this resolution is only of limited utility. Problems like the study of the width and profile of an acoustic Raman peak, ${ }^{8}$ the characterization of complex non-periodic structures through their Raman spectrum, ${ }^{9}$ or the analysis of the isotopic effects in gases ${ }^{10}$ and ultra-pure crystals $^{11,12}$ require a resolution close to $0.01 \mathrm{~cm}^{-1}$ or better. Other investigations that could benefit from higher resolution optical spectra include the study of donors/acceptors energies in semiconductors through photoluminescence, ${ }^{13,14}$ the analysis of anharmonic effects in optical phonons, ${ }^{15}$ and the characterization of single molecules via surface enhanced Raman scattering (SERS). ${ }^{16,17}$ This would normally involve the use of specific ultra-high resolution Raman spectroscopy systems, like double spectrometers of very long focal distance ${ }^{12,18}$ or one of several Fabry-Pérot (FP) ${ }^{19,20}$ and FP/spectrometer tandem configurations. ${ }^{21,22}$ However, although they can provide a very good resolution, the former can be expensive, have a small throughput and can be unstable, while the latter are slow and are usually conceived to measure small spectral windows.

a)grozas@cab.cnea.gov.ar
We present in this article a new ultra-high resolution Raman spectroscopy technique based in a Fabry-Pérot/triple spectrometer tandem with multichannel acquisition, which overcomes several of these problems. The system was developed specifically to measure low intensity, complex and spectrally extended acoustic Raman spectra. ${ }^{8}$ Accordingly, the design objective of the technique presented here was to obtain a resolution better than $0.01 \mathrm{~cm}^{-1}(1.25 \mu \mathrm{eV})$ without compromising the high sensitivity and the wide spectral window of a standard Raman spectrometer with multichannel acquisition. The key advance of this system with respect to previous FP/spectrometer tandems ${ }^{21,22}$ is its ability to follow simultaneously and independently the intensity of several transmission modes of the FP through the use of an extended multichannel detector. In this way, the long acquisition times required by a similar system with monochannel acquisition are reduced to a single measurement in which all the spectral ranges of interest are acquired in parallel.

In Sec. II we will describe the tandem system and its working principle. The procedures for energy calibration, tuning, and resolution estimation of the system will be detailed in Sec. III. Finally, in Sec. IV we will present some experimental results that show the capabilities of this new system for ultra-high resolution Raman scattering.

\section{THE FP/TRIPLE SPECTROMETER/CCD TANDEM}

As shown in Fig. 1, the system is composed of a singlepass Fabry-Pérot interferometer ${ }^{19}$ coupled to a triple spectrometer Dilor XY800 in additive configuration. The light to be analyzed is collected from the sample by the lens $\mathrm{L}_{\mathrm{col}}$, filtered through the FP, and then focused by the lens $\mathrm{L}_{\text {in }}$ into the entrance slit of the spectrometer. The FP contains two high quality $(\lambda / 200)$ dielectric mirrors for the near-infrared $(97 \%$ peak reflectivity centered either at 730 or $840 \mathrm{~nm}$ ), which are kept parallel at a fixed distance $d_{\mathrm{fp}}$ by three high quality cylindrical silica spacers (Table I). The diameter of the useful aperture of the system is $4 \mathrm{~cm}$, helping to the collection of low Raman signals. The mirrors are enclosed into a sealed 


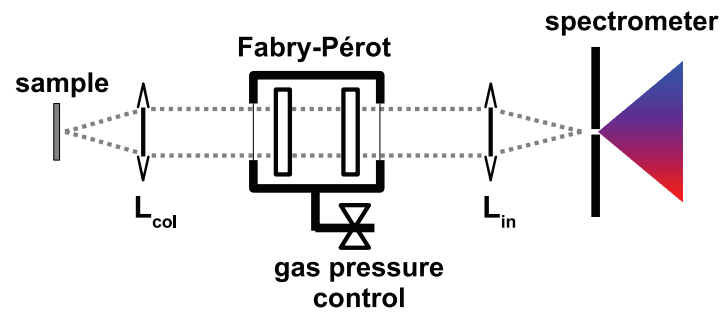

FIG. 1. Scheme of the Fabry-Pérot/spectrometer tandem. The light collected from the sample is filtered by the Fabry-Pérot interferometer before being analyzed by the triple spectrometer. Reprinted with permission from AIP Conf. Proc. 1199, 169 (2010). Copyright 2009 AIP Publishing LLC. ${ }^{31}$

chamber connected to a gas distribution and vacuum system, which allows for the control of the pressure and composition of the gas inside it. In all the experiments presented here the gas inside the FP chamber was pure nitrogen. The triple spectrometer is equipped with a Charge-Coupled Device (CCD) multichannel detector, cooled with liquid nitrogen down to $140 \mathrm{~K}$ to minimize thermal noise. This is specially important in view of the low signals to be acquired, typically of the order of 1 to 10 counts/s.

A FP interferometer has defined resonant modes due to the interference of the light reflected back and forth between both mirrors. When used as a light filter its transmission coefficient is given by the Airy function ${ }^{19}$

$$
T=\frac{T_{M}}{1+\frac{4 R}{(1-R)^{2}} \sin ^{2}\left(\frac{\phi}{2}\right)},
$$

with $T_{M}$ the maximum transmission, $R$ the reflectivity of each mirror (assumed equal in this example), and

$$
\phi=\frac{4 \pi n_{g} d_{\mathrm{fp}} \cos (\beta)}{\lambda_{L}} .
$$

Here $n_{g}$ is the refractive index of the gas between the mirrors, $d_{\mathrm{fp}}$ their separation, $\lambda_{L}$ the wavelength of light in vacuum, and $\beta$ the incidence angle. Based on the experimental conditions used we will consider from now on only normal incidence ( $\beta$ $=0$ ).

The function in Eq. (1) is essentially a series of peaks of quasi-Lorentzian profile with maxima at the wavelengths such that

$$
\phi=2 \pi m \Rightarrow \sigma_{L}(m)=\frac{1}{\lambda_{L}(m)}=\frac{m}{2 n_{g} d_{\mathrm{fp}}} .
$$

The energy separation between maxima, i.e., the free spectral range

$$
\mathrm{FSR}=\sigma_{L}(m+1)-\sigma_{L}(m)=\frac{1}{2 n_{g} d_{\mathrm{fp}}} \quad \forall m \in \mathbb{N}
$$

TABLE I. Description of the two sets of silica spacers used in this work. $d_{\mathrm{fp}}$ is the actual length of the spacers, which appears in Eq. (2). The free spectral range (FSR) is calculated from Eq. (4).

\begin{tabular}{lcc}
\hline \hline Nominal length $(\mathrm{cm})$ & $d_{\mathrm{fp}}(\mathrm{cm})$ & FSR in vacuum $\left(\mathrm{cm}^{-1}\right)$ \\
\hline 0.6 & $0.6011 \pm 0.0003$ & $0.8318 \pm 0.0004$ \\
1.0 & $1.0325 \pm 0.0005$ & $0.4843 \pm 0.0002$ \\
\hline \hline
\end{tabular}

of the FP, is a constant value dependent only on the distance $d_{\mathrm{fp}}$ and the refraction index $n_{g} .{ }^{23}$ For the experiments described here two sets of spacers of different sizes and, consequently, two different FSRs, were used (Table I). We will see latter in Sec. III C that the choice of $d_{\mathrm{fp}}$ results from a compromise between the resolution required by the experiment and the intrinsic resolution of the multichannel spectrometer.

The procedure to acquire a spectrum using the $\mathrm{FP} /$ spectrometer/CCD tandem is outlined in Fig. 2. Let us assume that the solid line in panel (a) is the real spectrum we want to measure. The vertical dotted lines show the central position of each pixel of the CCD, separated by $0.09 \mathrm{~cm}^{-1}$ (the total energy range shown is $1.7 \mathrm{~cm}^{-1}$ ). The resolution of the spectrometer, ideally $2-3$ pixels $\left(\sim 0.2-0.3 \mathrm{~cm}^{-1}\right)$, is clearly not enough to measure the real profile of the peak. If we collect the light coming from the sample and filter it through the FP, the spectrum we should theoretically obtain is the one simulated in panel (b) with a solid line: a series of peaks separated by the FSR, with the intensity of each peak modulated by the spectrum to be measured. The position of the FP transmission peaks will be determined by the refractive index $n_{g}$ of the gas inside the FP through Eq. (3). This index can be modified by varying the pressure of the gas, producing a linear shift in the energy of the peaks as a function of pressure (see Sec. III A below).

For the chosen values of $R$ and $d_{\mathrm{fp}}$, the width of the transmission peaks of the FP is much smaller than the resolution of the spectrometer (see Sec. III C), but the FSR is larger than this resolution. Under this condition, after it is acquired by the CCD the spectrum simulated in panel (b) results in the solid curve in (c): a series of peaks separated by the FSR but with a width that is given by the resolution of the spectrometer. ${ }^{24} \mathrm{As}$ long as the peaks can be distinguished in the measured spectrum, we can assign the total intensity of each peak, i.e., the shaded (white) area in (c), to the intensity transmitted through the corresponding mode of the FP, shown with the large full (empty) circle in (d). Plotting the total area below each peak as a function of the gas pressure, for example, we are then able to reconstruct the original spectrum from a series of measurements, as shown by the light dots in panel (d).

This simple example highlights one of the most important advantages of this method: for each gas pressure we obtain simultaneously as many points of the final spectrum as transmission peaks of the FP are inside the energy range measured by the CCD. Two peaks are shown in Fig. 2 for simplicity, but in a real situation this number is at least 10 and can be as large as 200 , depending on the experimental condition. We will see in Sec. III C that the resolution of a FP strongly depends on the FSR. In a system with monochannel acquisition, where only one peak at a time is measured, this implies a direct correlation between the total spectral range to be measured, the resolution, and the acquisition time. A doubling of the energy range, for example, requires either a doubling of the acquisition time (if two consecutive FSRs need to be measured) or a reduction to the half of the resolution (if a FSR twice larger is used). In the system presented here, it is enough to continuously shift the energy of the FP peaks by a single FSR (whatever its size) to measure the full spectrum at the desired resolution. When the spectra are complex, this disconnection 

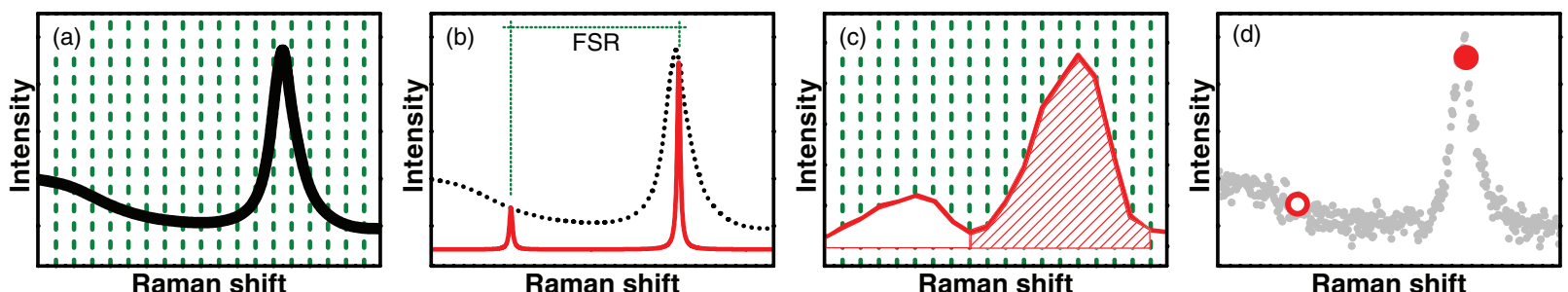

FIG. 2. Different stages on the reconstruction of the Raman spectrum using the FP/spectrometer/CCD tandem. Vertical dotted lines in (a) and (c) represent the position of the CCD pixels. The curve in (a) shows the real spectrum to be acquired, the solid line in (b) corresponds to the expected intensity after it has been filtered by the FP for a certain gas pressure. Panel (c) shows the filtered spectrum as measured by the spectrometer, the shaded (white) area corresponds to the full (empty) circle in (d). Changing the gas pressure allows for the acquisition of the full spectrum (light dots in (d)). Panels (c) and (d) correspond to real experimental data from Ref. 8 (16-period sample). All four panels have the same horizontal total energy scale of $1.7 \mathrm{~cm}^{-1}$ (see the text for details).

between the FSR (and hence, the resolution) and the total spectral range to be measured is a fundamental improvement with respect to similar methods. ${ }^{21,22}$

\section{CALIBRATION AND TUNING OF THE TANDEM}

The method presented here requires the ability to shift and track the energy of the FP transmission modes in order to reconstruct the full spectrum from each measured point. However, the resolution of the triple spectrometer is not enough to define the exact position of the modes with the required precision, and an external control variable (independently calibrated to an energy scale) must be used to recover the energy of each point of the spectrum. We will present in Secs. III A and III B two possible methods to control and shift the energy at which each data point is measured: to change the pressure of the gas inside the FP, shifting the transmission modes; and to tune the energy of the laser, shifting the Raman spectrum. In Sec. III C, we will discuss how the resolution of the tandem can be both estimated a priori and experimentally measured.

\section{A. Gas pressure tuning}

The standard method used to control the energy of the transmission modes in FP systems with big apertures and mirrors is to change the pressure of the gas inside the FP chamber. On these systems, precisely moving the mirrors to change $d_{\mathrm{fp}}$ is impractical. ${ }^{20-22}$ On the system presented here, the pressure is varied using a needle valve as a controllable leak between a high pressure reservoir and a vacuum buffer connected to the FP. The pressure is measured close to the FP chamber in order to minimize any flow impedance contribution. Although the relationship between energy and gas pressure is approximately linear, we will see that several conditions must be fulfilled for this to be valid.

Let us consider again Eq. (3), but now fixing the wavelength at a value $\lambda_{L}$. We would like to know for which pressure $p_{m}$ of the gas inside the FP the transmission mode $m$ is exactly at wavelength $\lambda_{L}$. If the refractive index of the gas is a function of wavelength, pressure, and temperature $T$ we will have that

$$
\begin{aligned}
& n_{g}\left(\lambda_{L}, p_{m+r}, T\right)-n_{g}\left(\lambda_{L}, p_{m}, T\right) \\
& \quad=(m+r) \frac{\lambda_{L}}{2 d_{\mathrm{fp}}}-m \frac{\lambda_{L}}{2 d_{\mathrm{fp}}}=r \frac{\lambda_{L}}{2 d_{\mathrm{fp}}}
\end{aligned}
$$

for two modes $m$ and $m+r$. The dependence of $n_{g}$ with pressure for a dry gas can be expressed in a very good approximation by ${ }^{25}$

$$
n_{g}\left(\lambda_{L}, p, T\right)-1=\left[n_{\mathrm{stp}}\left(\lambda_{L}\right)-1\right]\left[C_{1}(T) p+C_{2}(T) p^{2}\right],
$$

where $n_{\text {stp }}$ is the refractive index in standard conditions $\left(15^{\circ} \mathrm{C}\right.$ and $1 \mathrm{~atm}) . C_{1}$ and $C_{2}$ are the functions only of $T$ and depend on the particular gas, but for standard dry air $\left(78 \% \mathrm{~N}_{2}\right)$ close to room temperature they are such that $\left|C_{2} / C_{1}\right| p \lesssim 5$ $\times 10^{-4}$ for $p \lesssim 1 \mathrm{~atm}^{25}$ Under these conditions, the quadratic term can be neglected and

$$
\begin{aligned}
& n_{g}\left(\lambda_{L}, p_{m+r}, T\right)-n_{g}\left(\lambda_{L}, p_{m}, T\right) \\
& \quad \simeq\left[n_{\text {stp }}\left(\lambda_{L}\right)-1\right] C_{1}(T)\left(p_{m+r}-p_{m}\right),
\end{aligned}
$$

with an error lower than $0.05 \%$. Defining an effective constant

$$
A\left(\lambda_{L}, T\right)=\left[n_{\mathrm{stp}}\left(\lambda_{L}\right)-1\right] C_{1}(T),
$$

we finally obtain

$$
p_{m+r}-p_{m} \simeq r \frac{\lambda_{L}}{2 d_{\mathrm{fp}} A\left(\lambda_{L}, T\right)} .
$$

Following this equation, a way to measure the parameter $A$ is to send a laser of known wavelength through the FP interferometer, looking for the pressures $p_{m+r}$ at which the transmission reaches a maximum. Here, $m$ is an arbitrary number, usually unknown. Fig. 3 shows an example of this procedure for pure nitrogen at room temperature, using the spacers of nominal length $d_{\mathrm{fp}} \sim 0.6 \mathrm{~cm}$ (see Table I) and a laser with $\lambda_{L}$ $=(755.58 \pm 0.10) \mathrm{nm}$. From the slope of the curve we calculate in this case $A=(3.626 \pm 0.002) \times 10^{-7}$ Torr $^{-1}$. Notice that in this experiment what is changing is the optical length $d_{\mathrm{fp}} n_{g}$, and it is then necessary to know $d_{\mathrm{fp}}$ independently to obtain $A$.

Once we have measured $A$, we can go back to Eq. (3) and express the energy position of a transmission mode of the FP as a function of the pressure as

$$
\begin{aligned}
\Delta \sigma_{L}(m) & =-\left.\frac{m}{2\left(n_{g}^{0}\right)^{2} d_{\mathrm{fp}}} \frac{\partial n_{g}}{\partial p}\right|_{p_{0}} \Delta p+\cdots \\
& =-\frac{\sigma_{L}^{0}(m)}{1+A\left[\lambda_{L}^{0}(m), T\right] p_{0}} A\left[\lambda_{L}^{0}(m), T\right] \Delta p+\cdots,
\end{aligned}
$$




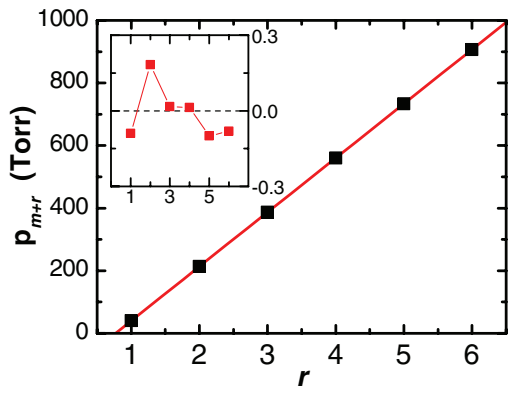

FIG. 3. Calibration of the $A\left(\lambda_{L}, T\right)$ parameter for pure nitrogen at room temperature, using $d_{\mathrm{fp}} \sim 0.6 \mathrm{~cm}$ and $\lambda_{L}=(755.58 \pm 0.10) \mathrm{nm}$. From the slope of the curve we calculate $A=(3.626 \pm 0.002) \times 10^{-7}$ Torr $^{-1}$. The inset shows the residuals of the linear fitting.

where $\Delta \sigma_{L}(m)=\sigma_{L}(m)-\sigma_{L}^{0}(m)$ and $\Delta p=p-p_{0} . \sigma_{L}^{0}(m)$ $=\lambda_{L}^{0}(m)^{-1}$ and $n_{g}^{0}=n_{g}\left[\lambda_{L}^{0}(m), p_{0}, T\right]$ are, respectively, the energy of the FP mode $m$ and the refraction index of the gas at a reference pressure $p_{0}$ (the average pressure during the experiment, for example). In a typical experiment $|\Delta p| \lesssim 100$ Torr, and it follows that the terms higher than linear can be disregarded as they introduce corrections that are at least an order of magnitude smaller than the precision with which $A$ can be measured. Assuming we stay in this linear regime, from the value of $A(756 \mathrm{~nm}, 290 \mathrm{~K})$ we obtained above it follows that

$$
\frac{\Delta \sigma_{L}(m)}{\Delta p}=(4.798 \pm 0.003) \times 10^{-3} \mathrm{~cm}^{-1} / \text { Torr }
$$

for an experiment performed around 500 Torr, at $756 \mathrm{~nm}$ and room temperature. Notice that, according to Eq. (10), this calibration is independent of $d_{\mathrm{fp}}$.

We will finish this section by looking into the energy and temperature dependence of $A$. Table II shows the refractive index of pure nitrogen in standard conditions for near-infrared wavelengths, and Table III shows the same index as a function of temperature at $800 \mathrm{~nm}$, both obtained from Ref. 26. According to this data and Eq. (8), the change in $A$ across the full wavelength range should be of the order of $0.5 \%$, and the change with temperature of the order of $0.3 \% /{ }^{\circ} \mathrm{C}$ near room temperature. This means that in a typical experiment, where the energy range acquired is of the order of 10 to $100 \mathrm{~cm}^{-1}$ ( $\lesssim 5 \mathrm{~nm}$ ), the wavelength dependence of $A$ can be disregarded and an average value $A\left(\lambda_{L}^{0}, T\right)$ can be used in Eq. (10) for all the FP modes $m$ in the range considered. The temperature dependence, on the other hand, means that a precise control of the temperature of the gas is needed to maintain the precision of the calibration during (and between) long experiments.

TABLE II. Wavelength dependence of the refractive index of pure nitrogen in standard conditions $\left(15^{\circ} \mathrm{C}\right.$ and $\left.1 \mathrm{~atm}\right)$. Data taken from Ref. 26.

\begin{tabular}{lc}
\hline \hline$\lambda_{L}(\mathrm{~nm})$ & {$\left[n_{\mathrm{stp}}\left(\lambda_{L}\right)-1\right]\left(\times 10^{-4}\right)$} \\
\hline 700 & 2.815 \\
800 & 2.808 \\
950 & 2.801 \\
\hline \hline
\end{tabular}

TABLE III. Temperature dependence of the refractive index of pure nitrogen at $800 \mathrm{~nm}$ and $1 \mathrm{~atm}$. Data taken from Ref. 26.

\begin{tabular}{lc}
\hline \hline Temperature $\left({ }^{\circ} \mathrm{C}\right)$ & {$\left[n_{g}(T)-1\right]\left(\times 10^{-4}\right)$} \\
\hline 15 & 2.808 \\
20 & 2.760 \\
25 & 2.713 \\
\hline \hline
\end{tabular}

\section{B. Laser tuning}

In the gas tuning method described above the energy of the spectrum to be measured is fixed, and the energy of the transmission peaks of the FP is shifted with respect to it. This method can be applied to measure any kind of optical spectrum. In the case of Raman scattering experiments, however, there is an alternative option: to shift the energy of the excitation laser while keeping the transmission peaks at a fixed energy. The final result would be similar: a different point of the spectrum would be acquired for each energy of the laser, and the full spectrum can be reconstructed after changing the energy of the laser by a FSR, as we described in Sec. II.

A prerequisite for this laser tuning method to work is the capability to precisely control the wavelength of the excitation laser. However, the developments in the past decade in the area of single mode tunable laser diodes and stabilized ring lasers have made this a relatively simple task. We tested this method using a near-infrared Ti:Sapphire single mode Spectra-Physics Matisse TS ring laser, the wavelength of which can be locked to an external confocal cavity with a precision better than $2 \times 10^{-6} \mathrm{~cm}^{-1}$. The energy of this reference cavity, in turn, can be modified by applying a voltage to the piezoelectric actuator that changes the distance between its mirrors. Controlling this external voltage, this system allows us to shift the laser energy by as much as $1.6 \mathrm{~cm}^{-1}$, with a precision of the order of $10^{-4} \mathrm{~cm}^{-1}$.

As the relationship between the voltage applied to the external reference cavity and the laser energy is linear, the calibration of the energy scale in this method is reduced to simply measure the FSR of the FP in units of this control variable. Keeping the gas pressure $p$ and temperature $T$ constant, the transmission of the laser through the FP can be monitored to find the voltage $V(m)$ at which the laser energy matches the energy of the mode $m$. Following Eq. (4), we can define a conversion constant

$$
\begin{aligned}
B\left(\lambda_{L}, p, T\right) & =\frac{\mathrm{FSR}}{V(m+1)-V(m)} \\
& =\frac{1}{2 n_{g} d_{\mathrm{fp}}[V(m+1)-V(m)]} .
\end{aligned}
$$

The dependence of $B$ on the wavelength $\lambda_{L}$, the temperature $T$, and the pressure $p$ enters this equation through the refraction index $n_{g}$ of the gas inside the FP. For a certain change $\Delta V$ on the voltage, the shift in laser energy will be then

$$
\Delta \sigma_{L}=B\left(\lambda_{L}^{0}, p, T\right) \Delta V
$$

where $\lambda_{L}^{0}$ is the average laser wavelength during the experiment. 


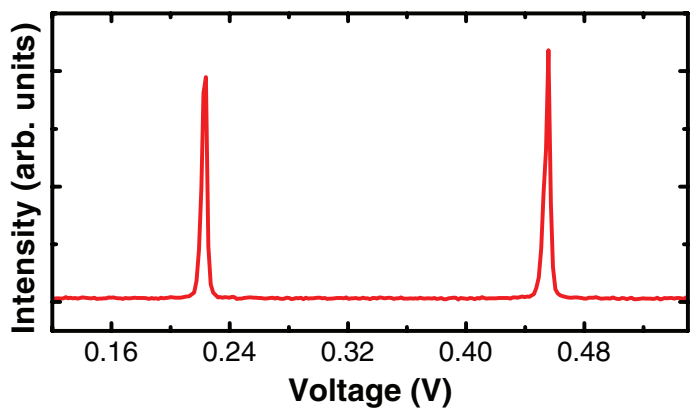

FIG. 4. Laser intensity transmitted through the FP as a function of the voltage applied to the external reference cavity of the Spectra-Physics Matisse TS laser. Acquired at room temperature, $780 \mathrm{~nm}, 575$ Torr, and using $d_{\mathrm{fp}} \sim 0.6 \mathrm{~cm}$ spacers. The separation between both transmission modes is $(232.1 \pm 0.3) \mathrm{mV}$, resulting in a calibration coefficient $B=(3.591 \pm$ $0.005) \mathrm{cm}^{-1} / \mathrm{V}$.

Figure 4 shows, as an example, the transmission of the FP with $d_{\mathrm{fp}} \sim 0.6 \mathrm{~cm}$ spacers measured using the SpectraPhysics Matisse TS laser, at a constant pressure of 575 Torr and room temperature. The voltage on the reference cavity was changed a total $0.5 \mathrm{~V}$ following a linear ramp of $0.001 \mathrm{mV} / \mathrm{s}$, and the transmitted intensity was monitored in the triple spectrometer $\mathrm{CCD}$ as a function of time. Each of the intensity peaks corresponds to a different FP mode, and the distance between them is (232.1 $\pm 0.3) \mathrm{mV}$. From Eq. (11), this represents a conversion constant $B(780 \mathrm{~nm}, 575$ Torr, $293 \mathrm{~K})=(3.591 \pm 0.005) \mathrm{cm}^{-1} / \mathrm{V}$.

The ideal case for an experiment performed using this method is when the FP is in vacuum. In this case, $n_{g}$ is 1 and $B$ becomes independent of $\lambda_{L}^{0}$ and $T$. However, in practice this independence is maintained even when $p>0$ : the refraction index data shown in Tables II and III correspond to changes on $B$ of the order of $0.0001 \%$ over a $200 \mathrm{~nm}$ range and $0.0001 \% /{ }^{\circ} \mathrm{C}$ near room temperature, variations that are much smaller than the precision with which $B$ is measured. This allows to use a single calibration coefficient for all the wavelengths and temperatures, provided a precise control of the pressure of the gas is present. ${ }^{27}$

The second main advantage of this method with respect to the gas tuning method presented before is that the laser energy can be controlled more easily than the gas pressure, allowing more flexibility in the measurements. For example, the laser energy could be moved back and forth automatically between two limits in order to average multiple measurements. Another option could be to shift the laser in discrete steps, integrating for an arbitrary length of time at each step. Both these scenarios would increase the signal to noise ratio of the final spectrum, and would be more difficult to implement by gas pressure control alone. ${ }^{28}$

Finally, this method has at least two disadvantages. On the one hand, it requires extra hardware in the form of a (usually) more expensive laser. On the other hand, as the laser energy is changing from point to point of the spectrum this method cannot be used in Raman resonant conditions, unless the width of the resonance is much larger than the total shift of the laser (typically 1-2 times the FSR).

\section{Resolution}

There are several factors that should be taken into account to define the resolution of the tandem for Raman scattering experiments. First, the transmission modes of the FP have a finite linewidth $\Delta \sigma_{f p}$ defined by the reflectivity of the mirrors, their surface quality, and relative separation and alignment. Second, the linewidth of the laser itself can make an important contribution. And finally, experimental aspects as the acquisition time per spectrum point and the rate of change of the gas pressure or laser energy can add to the final result if not chosen appropriately.

The transmission coefficient described in Eq. (1) corresponds to an ideal FP. In this case, the full-width at halfmaximum (FWHM) of the transmission modes is given only by the reflectivity of the mirrors and the distance $d_{\mathrm{fp}}$ between them, ${ }^{19}$ contributing to the final resolution with

$$
\Delta \sigma_{R} \simeq \frac{1-R}{\pi \sqrt{R}} \mathrm{FSR}
$$

for $R \sim 1$. For a reflectivity of $97 \%$ this width is $\sim 1 \%$ of the FSR.

$\Delta \sigma_{R}$ can be interpreted as an homogeneous broadening. In a real system, however, the distance between the mirrors will vary from point to point due to imperfections on their surfaces, resulting in a superposition of interference patterns slightly shifted in energy (i.e., an inhomogeneous broadening). If we consider only the linewidth produced by this effect it will be proportional to the relative change in $d_{\mathrm{fp}},{ }^{19}$ resulting

$$
\Delta \sigma_{D} \simeq \frac{2 \Delta d}{\lambda_{L}} \mathrm{FSR} .
$$

$\Delta d$ is the typical size of the surface defects, of the order of $\lambda_{L} / 100$ or less for custom made interferometry mirrors.

The last important factor affecting the linewidth of the FP modes is the angle of incidence. Even if we set $\beta=0$, the finite size of the light source and the aberrations of the lenses determine a range of incident angles $\Delta \beta$ for a single wavelength. This introduces again an energy shift of the interference pattern which is translated into a linewidth of ${ }^{19}$

$$
\Delta \sigma_{A} \simeq \frac{\Delta \beta^{2}}{2 \lambda_{L}}
$$

For example, in the Raman experiments presented here the typical diameter of the laser spot is $50 \mu \mathrm{m}$. Using a lens of $8 \mathrm{~cm}$ focal length to collect the scattered light the apparent angle subtended by this spot is $\Delta \beta \sim 6 \times 10^{-4}$. The resulting mode linewidth at $\lambda_{L}=840 \mathrm{~nm}$ is $\Delta \sigma_{A} \sim 2.3 \times 10^{-3} \mathrm{~cm}^{-1}$, at least two times smaller than the other contributions when FSR $\gtrsim 0.5 \mathrm{~cm}^{-1}$. The depth of field of the collection lens, which should also add to this apparent angle, is irrelevant in this case due to the very small depth $(<1 \mu \mathrm{m})$ of the active zone of the samples under study.

The exact profile and linewidth of the transmission peaks cannot be known in advance without a detailed knowledge of the surface defects of the mirrors, but it can be expected that $\Delta \sigma_{f p}$ will be larger than any of the three contributions and smaller than their sum. ${ }^{19}$ The theoretical values of these contributions for the two FP spacers used in this work are shown in Table IV, along with the maximum expected value 
TABLE IV. Theoretical and experimental values of the different contributions to the final resolution, including both the FP and laser linewidths, for the two tandem configurations used in Sec. IV of this work (see text for further details and definitions). $\Delta \sigma$ is given in $10^{-3} \mathrm{~cm}^{-1}$, and the theoretical values are calculated for the central wavelength $\lambda_{L}=840 \mathrm{~nm}$ of one of the sets of mirrors. The single line Coherent laser used has a nominal linewidth $\Delta \sigma_{\text {las }}^{\text {nom }} \sim 2.5 \times 10^{-6} \mathrm{~cm}^{-1}$, experimentally negligible.

\begin{tabular}{|c|c|c|c|c|c|c|c|c|c|c|c|}
\hline \multirow[b]{2}{*}{ Config. } & \multirow[b]{2}{*}{ Spacers $(\mathrm{cm})$} & \multicolumn{5}{|c|}{ FP } & & \multicolumn{2}{|c|}{ Laser } & \multicolumn{2}{|c|}{ Total experimental } \\
\hline & & $\Delta \sigma_{R}$ & $\Delta \sigma_{D}$ & $\Delta \sigma_{A}$ & $\Delta \sigma_{f p}^{\max }$ & $\Delta \sigma_{f p}^{\exp }$ & & $\Delta \sigma_{\text {las }}^{\text {nom }}$ & $\Delta \sigma_{\text {las }}^{\exp }$ & $\Delta \sigma_{\text {total }}^{\exp }$ & $\mathcal{F}$ \\
\hline A & 0.6 & 8.1 & 8.3 & 2.3 & 18.7 & $10 \pm 2$ & $\mathrm{SP}^{\mathrm{a}}$ & 13.2 & $14.3 \pm 1.7$ & $20.5 \pm 1.1$ & $41 \pm 3$ \\
\hline $\mathrm{B}$ & 1.0 & 4.7 & 4.8 & 2.3 & 11.8 & $8.2 \pm 0.9$ & $\mathrm{Co}^{\mathrm{b}}$ & $\ldots$ & $\ldots$ & $8.2 \pm 0.9$ & $59 \pm 6$ \\
\hline
\end{tabular}

${ }^{a}$ Spectra-Physics 3900S (standing wave laser).

${ }^{\mathrm{b}}$ Coherent MBR-110 (stabilized ring laser).

for $\Delta \sigma_{f p}$, denoted by $\Delta \sigma_{f p}^{\max }$. In a first approximation (disregarding $\Delta \sigma_{A}$ ) the FP resolution results proportional to the FSR. Consequently, the selection of $d_{\mathrm{fp}}$ is dictated by a balance between maximizing the resolution (smaller FSR) and being able to separate the transmission modes with the spectrometer (larger FSR). This balance will result in an increase of the resolution of the tandem by a factor of the order of $\mathrm{FSR} / \Delta \sigma_{f p}$ with respect to the spectrometer alone (around 45 for the FP system used here).

Regarding the laser contribution, for the experiments presented in Sec. IV we used two different near-infrared Ti:Sapphire lasers: a Spectra-Physics 3900S standing wave laser with two intra-cavity etalons, and a stabilized single mode Coherent MBR-110 ring laser. The former has a nominal linewidth of $\Delta \sigma_{\text {las }}^{\text {nom }} \sim 13 \times 10^{-3} \mathrm{~cm}^{-1}$, corresponding to 2 or 3 longitudinal modes of the laser cavity, while the latter has a linewidth of $2.5 \times 10^{-6} \mathrm{~cm}^{-1}$ and can be considered as an energy delta function. These lasers were combined with the two FP spacers in order to present two resolution situations that are clearly different (see Table IV). Configuration A is a "low" resolution configuration, with a large FSR that allows to easily separate the contributions of the different FP modes on the CCD. The optical construction of the standing wave laser is also more versatile, facilitating energy dependent experiments that require big wavelength changes. The "high" resolution configuration $\mathrm{B}$, on the other hand, has a smaller FSR that is at the limit of the resolution of the $\mathrm{CCD}$, and has a negligible laser linewidth contribution, showing the maximum resolution that can be achieved with the system.

From an experimental point of view, once the energy scale is calibrated as described in Secs. III A and III B, the real resolution of the Raman system is obtained by measuring with the tandem the intensity profile of the laser. The result of this measurement will be the convolution of the profile of a FP transmission mode and the profile of the laser, and will determine the resolution that can be obtained with that particular laser-tandem combination in a Raman scattering experiment.

As an example, Fig. 5 shows the profile of the SpectraPhysics $3900 \mathrm{~S}$ laser at $755 \mathrm{~nm}$, measured with the tandem using the $d_{\mathrm{fp}} \sim 0.6 \mathrm{~cm}$ spacers (full circles). The experimental linewidth of the peak is in this case $\Delta \sigma_{\text {total }}^{\text {exp }}=(0.0187$ $\pm 0.0008) \mathrm{cm}^{-1}$. The peak can be accurately reproduced by a Voigt profile (full line). If we associate the Lorentzian component of the fit to the FP contribution and the Gaussian compo- nent to the laser contribution, we can estimate their linewidths to be $\Delta \sigma_{f p}^{\text {exp }}=0.010 \mathrm{~cm}^{-1}$ and $\Delta \sigma_{\text {las }}^{\text {exp }}=0.012 \mathrm{~cm}^{-1}$, respectively. Table IV shows the results of repeating this laser fitting procedure under different conditions along one month of Raman scattering experiments. $\Delta \sigma_{f p}^{\text {exp }}, \Delta \sigma_{\text {las }}^{\text {exp }}$, and $\Delta \sigma_{\text {total }}^{\text {exp }}$ are averaged over 19 measurements in the case of configuration $\mathrm{A}$, and over 11 measurements in the case of configuration B. In the first case the total linewidth is consistent with a combination of the laser and tandem contributions, while in the second case the fitted profiles are clearly Lorentzian, as expected if the linewidth is produced only by the FP.

The average values for the total linewidth $\Delta \sigma_{\text {total }}^{\exp }$ and the finesse $\mathcal{F}=\mathrm{FSR} / \Delta \sigma_{\text {total }}^{\text {exp }}$ can be taken as a good estimation of the typical resolution of each of the laser-tandem combinations for Raman scattering measurements. However, as either the FP modes energies (gas tuning) or the laser energy (laser tuning) follow a linear dependence with time during the experiment, the CCD acquisition time per spectrum point and the resulting discretization of the energy scale should also be taken into account when considering the final resolution.

Finally, we can define an effective finesse for the $\mathrm{FP} /$ spectrometer/CCD tandem given by the relation between the total spectral range acquired in a single experiment (several FSRs) and $\Delta \sigma_{\text {total }}^{\text {exp }}$. This number will typically be of the order of 10 to 100 times larger than the value of $\mathcal{F}$ presented in Table IV, and it is a good representation of the advantage of this system over standard tandems with monochannel acquisition.

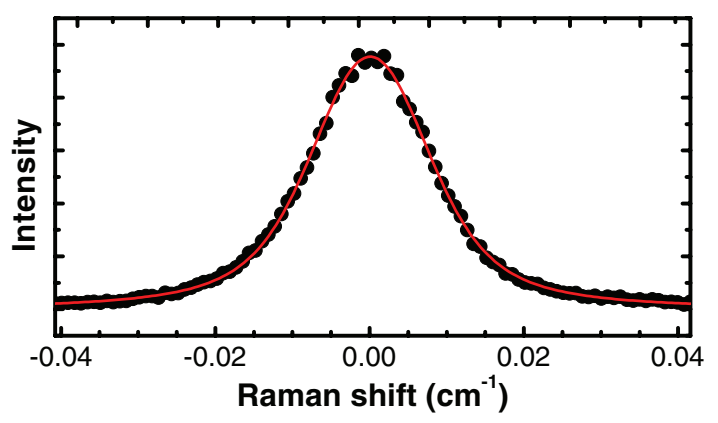

FIG. 5. Experimental profile of the Spectra-Physics 3900 S laser acquired at $755 \mathrm{~nm}$ and with the $d_{\mathrm{fp}} \sim 0.6 \mathrm{~cm}$ spacers (full circles). The fitting (full line) corresponds to a Voigt profile with a total width of $(0.0187 \pm 0.0008) \mathrm{cm}^{-1}$. Reprinted with permission from AIP Conf. Proc. 1199, 169 (2010). Copyright 2009 AIP Publishing LLC. ${ }^{31}$ 


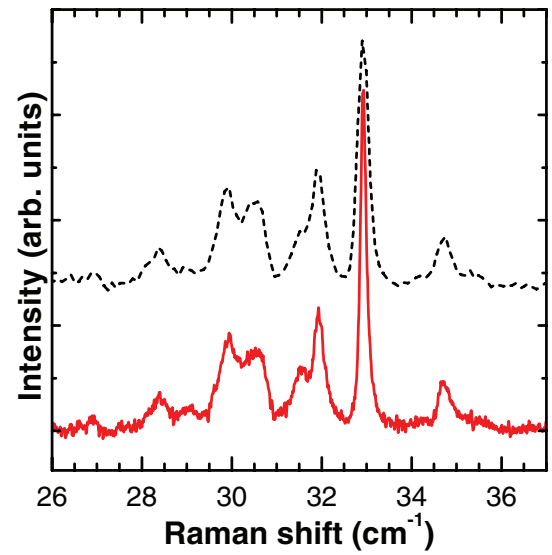

FIG. 6. Raman scattering spectrum of a $1 \mathrm{THz}$ semiconductor acoustic cavity acquired with the spectrometer alone (dashed line) and with the tandem (solid line). Data from Ref. 8 (12-period sample).

\section{EXPERIMENTAL TEST}

This new technique was originally developed specifically for the ultra-high resolution Raman scattering experiments presented in Ref. 8, and has been used to study the lifetime and mode broadening of planar semiconductor acoustic cavities ${ }^{29}$ for the last few years. ${ }^{8,30}$ We will present here two examples of these results to show the capabilities of the $\mathrm{FP} /$ triple spectrometer/CCD tandem regarding energy range and resolution. Both examples were acquired using the gas pressure tuning method.

For the first example, we present in Fig. 6 a comparison of the Raman spectrum of a $1 \mathrm{THz}$ semiconductor acoustic cavity acquired both with a high resolution triple spectrometer (dashed line) and with the tandem (solid line). The details of the sample and acoustic phonons Raman peaks are outside the scope of this paper and can be found in Ref. 8 (12-period sample). The wavelength of the laser, a Spectra-Physics 3900S, was set at $756 \mathrm{~nm}$. The FP interferometer was fitted with mirrors centered at $730 \mathrm{~nm}$ and $d_{\mathrm{fp}} \sim 0.6 \mathrm{~cm}$ spacers, and the gas pressure change rate was around $1.4 \mathrm{Torr} / \mathrm{min}$ (total change of 145 Torr, centered at 475 Torr). The experimental resolution of the full system including the laser was $0.02 \mathrm{~cm}^{-1}$, with a FSR of $0.83 \mathrm{~cm}^{-1}$ (configuration A in Table IV). The higher resolution of the tandem allowed in this case to determine that the only resolution-limited peak in the top spectrum is the one located at $33 \mathrm{~cm}^{-1}$ (the acoustic cavity mode), which reduces from a linewidth of $0.30 \mathrm{~cm}^{-1}$ with the spectrometer to its real linewidth of $0.16 \mathrm{~cm}^{-1}$ as measured with the tandem.

Figure 6 shows only the central part of the total $27 \mathrm{~cm}^{-1}$ energy range acquired in the experiment. This corresponds to approximately 33 times the FSR, representing an effective finesse of $\sim 1350$ for this measurement. The size of this energy range is limited by the triple spectrometer configuration and its laser rejection capabilities, and it is not related to the tandem technique itself. Essentially, if a certain energy range can be measured using the spectrometer, exactly the same range can be measured at a much higher resolution using the tan$\mathrm{dem}$. The tradeoff to this increased resolution is the longer acquisition time because of the lower throughput and the se-

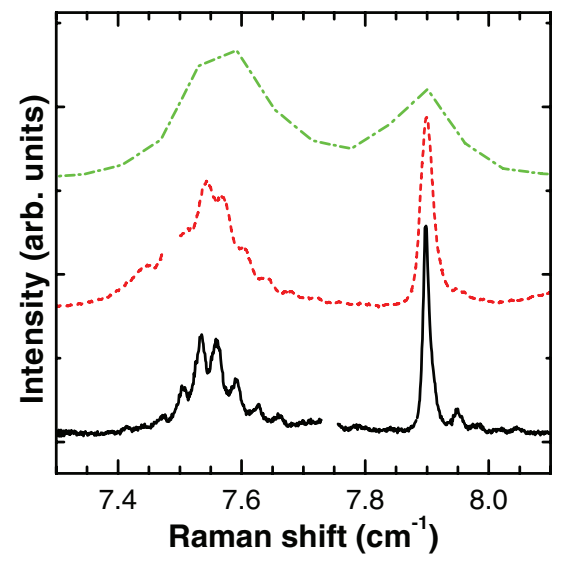

FIG. 7. Raman scattering spectrum of a $250 \mathrm{GHz}$ semiconductor acoustic cavity acquired with the spectrometer alone (dashed-dotted line), the tandem with $d_{\mathrm{fp}} \sim 0.6 \mathrm{~cm}$ spacers and a standing wave laser (dashed line), and the tandem with $d_{\mathrm{fp}} \sim 1.0 \mathrm{~cm}$ spacers and a single line ring laser (solid line). The resolutions for each experiment are $0.19 \mathrm{~cm}^{-1}, 0.02 \mathrm{~cm}^{-1}$, and $0.008 \mathrm{~cm}^{-1}$, respectively.

quential nature of the measurement. In this case, the spectrum taken with the spectrometer required only $10 \mathrm{~s}$, while the tandem spectrum is composed of 60 acquisitions of $120 \mathrm{~s}$ each ( $\sim 2 \mathrm{~h}$ total). A traditional tandem system with monochannel acquisition, however, would have taken close to $66 \mathrm{~h}$ to obtain the same spectrum at an equivalent resolution.

The second example, presented in Fig. 7, allows to gauge the maximum resolution that can be achieved with this system. The three Raman scattering spectra correspond to a $250 \mathrm{GHz}$ semiconductor acoustic cavity (details on the sample and Raman spectrum will be published elsewhere). All three spectra were taken at $856 \mathrm{~nm}$, each one at a different resolution. The top spectrum (dashed-dotted line) was acquired with the triple spectrometer at its highest possible resolution in additive configuration $\left(0.19 \mathrm{~cm}^{-1}\right)$, while the other two were acquired with the tandem using two different configurations. For the middle spectrum (dashed line), the FP was fitted with the $d_{\mathrm{fp}} \sim 0.6 \mathrm{~cm}$ spacers and the laser used was the SpectraPhysics $3900 \mathrm{~S}\left(0.02 \mathrm{~cm}^{-1}\right.$ resolution and FSR of $\left.0.83 \mathrm{~cm}^{-1}\right)$. For the bottom spectrum (solid line), $d_{\mathrm{fp}} \sim 1.0 \mathrm{~cm}$ spacers and the single mode Coherent MBR-110 laser were used $\left(0.008 \mathrm{~cm}^{-1}\right.$ resolution and FSR of $\left.0.48 \mathrm{~cm}^{-1}\right)$. In both cases the FP mirrors were centered at $840 \mathrm{~nm}$. These two configurations correspond to configurations $\mathrm{A}$ and $\mathrm{B}$ in Table IV, respectively. The total energy range acquired was only $2.2 \mathrm{~cm}^{-1}$ due to the strong stray-light coming from the laser at such low Raman shifts ( $\sim 6$ times the FSR at the highest resolution).

While the spectrum from the triple spectrometer shows only two featureless resolution-limited peaks, the two other spectra show details on the peak profiles that can only be identified thanks to the extra resolution provided by the tandem. On the one hand, the lower energy peak shows intensity oscillations, which start to be visible in the middle spectrum and are very clear in the bottom one. These oscillations are related to phonon interference effects and allow us to estimate a minimum coherence length for $250 \mathrm{GHz}$ phonons. The high energy peak, on the other hand, corresponds to the acoustic cavity mode and its linewidth is directly related to 
its lifetime. This peak is still resolution limited in the central spectrum (linewidth of $0.025 \mathrm{~cm}^{-1}$ ), and only with the highest resolution configuration the linewidth and lifetime of the mode can be extracted reliably. In this latter case the FWHM of the peak is $0.012 \mathrm{~cm}^{-1}$, and after detailed Raman simulations and a deconvolution of the experimental resolution component we can estimate its true linewidth to be $(0.0064$ $\pm 0.0013) \mathrm{cm}^{-1}$.

Finally, it is important to notice that even if the tandem requires much more time to acquire the Raman spectrum in comparison with the spectrometer alone, the acquisition time is generally independent of the spacer-defined resolution $\left(\Delta \sigma_{R}\right.$ and $\left.\Delta \sigma_{D}\right)$. This is achieved by selecting different rates of gas pressure change to compensate for the different FSRs, in this case of $\sim 3.6 \mathrm{Torr} / \mathrm{min}$ for the lower resolution configuration (total change of 235 Torr) and $\sim 1.9$ Torr/min for the higher resolution configuration (total change of 125 Torr). In both cases the spectrum is composed of 660 measurements of $5 \mathrm{~s}$ each, for a total acquisition time of $\sim 1 \mathrm{~h}$.

\section{CONCLUSIONS}

The acquisition of very high resolution Raman spectra $\left(\sim 0.01 \mathrm{~cm}^{-1}\right)$ is a problem that requires of very specific tools. We have presented here a new ultra-high resolution Raman spectroscopy technique that is specially tailored for low intensity, complex and spectrally extended spectra, based in a Fabry-Pérot/triple spectrometer tandem with multichannel acquisition.

First, we described the technique and detailed the working principle of the system. We showed that the addition of a CCD multichannel detector in conjunction with a high resolution triple spectrometer dramatically reduces the long acquisition times required by similar tandem systems with monochannel detectors. This is made possible by our system's ability to independently record the intensity of several FabryPérot transmission modes in a simultaneous way.

Second, we characterized the tandem system in terms of energy calibration and resolution, both theoretically and experimentally. In particular, we proposed two different methods for the control of the energy at which each experimental point is acquired, namely, to change the gas pressure inside the FP and to change the energy of the laser. We compared their advantages and disadvantages, and made explicit the limitations of the energy calibration. These results are not exclusive to the system presented here and can be applied to any FP/spectrometer tandem.

Finally, we presented experimental results that provide evidence of the capabilities of this system for ultra-high resolution Raman scattering. The system increases the resolution of a typical Raman spectrometer by a factor of 40 or more without affecting the spectral range, at the expenses of longer acquisition times. These examples also demonstrate that this technique can offer the same resolution than other tandem systems with monochannel acquisition but with a much wider spectral range, providing an effective finesse of the order of 1000 or more.

\section{ACKNOWLEDGMENTS}

The authors would like to acknowledge R. Oullion (UPMC, Paris) for his help in the initial setup of the FP, and A. Lemaitre (LPN, Marcoussis) for growing the samples used for the experiments in Sec. IV.

${ }^{1}$ B. Jusserand, and M. Cardona, "Topics in applied physics," in Light Scattering in Solids V: Superlattices and Other Microstructures, edited by M. Cardona and G. Güntherodt (Springer-Verlag, Berlin, 1989), Vol. 66, Chap. III, pp. 49-152; R. Merlin, ibid., Chap. V, pp. 214-232; M. H. Grimsditch, ibid., Chap. VII, pp. 285-302.

${ }^{2}$ T. Ruf, "Phonon Raman scattering in semiconductors, quantum wells and superlattices: Basic results and applications," in Springer Tracts in Modern Physics (Springer-Verlag, Berlin, 1998), Vol. 142.

${ }^{3}$ A. Fainstein, and B. Jusserand, "Topics in applied physics," in Light Scattering in Solids IX: Novel Materials and Techniques, edited by M. Cardona and R. Merlin (Springer-Verlag, Berlin, 2007), Vol. 108, Chap. II, pp. 17114; A. Mlayah and J. Groenen, ibid., Chap. IV, pp. 237-316.

${ }^{4}$ P. Lacharmoise, A. Fainstein, B. Jusserand, and V. Thierry-Mieg, Appl. Phys. Lett. 84, 3274 (2004).

${ }^{5}$ G. Rozas, M. F. Pascual Winter, A. Fainstein, B. Jusserand, P. O. Vaccaro, and S. Saravanan, Phys. Rev. B 77, 165314 (2008).

${ }^{6}$ A. Bruchhausen, A. Fainstein, A. Soukiassian, D. G. Schlom, X. X. Xi, M. Bernhagen, P. Reiche, and R. Uecker, Phys. Rev. Lett. 101, 197402 (2008).

${ }^{7}$ N. D. Lanzillotti-Kimura, A. Fainstein, B. Jusserand, and A. Lemaître, Phys. Rev. B 79, 035404 (2009).

${ }^{8}$ G. Rozas, M. F. Pascual Winter, B. Jusserand, A. Fainstein, B. Perrin, E. Semenova, and A. Lemaître, Phys. Rev. Lett. 102, 015502 (2009).

${ }^{9}$ N. D. Lanzillotti-Kimura, A. Fainstein, B. Jusserand, A. Lemaître, O. Mauguin, and L. Largeau, Phys. Rev. B 76, 174301 (2007).

${ }^{10}$ B. P. Stoicheff, Canadian J. Phys. 35, 730 (1957).

${ }^{11}$ H. D. Fuchs, C. H. Grein, R. I. Devlen, J. Kuhl, and M. Cardona, Phys. Rev. B 44, 8633 (1991).

${ }^{12}$ J. M. Zhang, M. Giehler, A. Göbel, T. Ruf, M. Cardona, E. E. Haller, and K. Itoh, Phys. Rev. B 57, 1348 (1998).

${ }^{13}$ A. K. Ramdas and S. Rodriguez, Rep. Prog. Phys. 44, 1297 (1981).

${ }^{14}$ E. S. Kumar, I. P. Anderson, Z. Deng, F. Mohammadbeigi, T. Wintschel, D. Huang, and S. P. Watkins, Semicond. Sci. Technol. 28, 045014 (2013).

${ }^{15}$ G. Lang, K. Karch, M. Schmitt, P. Pavone, A. P. Mayer, R. K. Wehner, and D. Strauch, Phys. Rev. B 59, 6182 (1999).

${ }^{16}$ P. G. Etchegoin and E. C. Le Ru, Anal. Chem. 82, 2888 (2010).

${ }^{17}$ E. Cortés, P. G. Etchegoin, E. C. Le Ru, A. Fainstein, M. E. Vela, and R. C. Salvarezza, J. Am. Chem. Soc. 135, 2809 (2013).

${ }^{18}$ J. P. Pinan, R. Ouillon, P. Ranson, M. Becucci, and S. Califano, J. Chem. Phys. 109, 5469 (1998).

${ }^{19} \mathrm{P}$. Bousquet, Spectroscopie Instrumentale (Dunod Université, Paris, 1969); M. Born and E. Wolf, Principles of Optics: Electromagnetic Theory of Propagation, Interference and Diffraction of Light, 7th ed. (Cambridge University Press, Cambridge, 2002).

${ }^{20}$ S. M. Lindsay, M. W. Anderson, and J. R. Sandercock, Rev. Sci. Instrum. 52, 1478 (1981).

${ }^{21}$ P. Ranson, R. Ouillon, and S. Califano, Chem. Phys. 86, 115 (1984).

${ }^{22}$ G. Pratesi and F. Barocchi, Measurement Science and Technology 6, 41 (1995).

${ }^{23}$ Strictly speaking, this expression for the FSR is an approximation, as the refraction index changes with wavelength and $n_{g}$ is different at modes $m$ and $m+1$. However, this change is so small for normal gases (of the order of $10^{-8} \mathrm{~nm}^{-1}$ in the near-infrared ${ }^{26}$ ) that can be completely ignored.

${ }^{24}$ To increase the amount of light that reaches the CCD it is convenient to increase the size of the entrance slit of the triple spectrometer. This decreases the spectrometer resolution, which will be normally lower than the ideal 2-3 pixels. However, this has no effect on the overall resolution of the system as long as the individual transmission peaks of the FP can be distinguished in the measured spectrum.

${ }^{25}$ K. P. Birch and M. J. Downs, Metrologia 30, 155 (1993); 31, 315 (1994).

${ }^{26}$ E. R. Peck and B. N. Khanna, J. Optical Society of America 56, 1059 (1966).

${ }^{27}$ Contrary to the gas tuning method, the calibration depends here on $n_{g}$ instead of $\left(n_{g}-1\right)$, making it much more robust to the same absolute changes in $n_{g}$ described by Tables II and III. However, some form of temperature control is still needed during the experiment: the absolute 
energy of the transmission peaks will shift with temperature to the order of $0.01 \mathrm{~cm}^{-1} /{ }^{\circ} \mathrm{C}$, even if FSR and $B$ stay almost constant. We are also assuming that the relation between voltage and laser energy stays constant along the whole wavelength range.

${ }^{28}$ Notice that there is a practical limit to how slowly the gas pressure can be changed, given by the minimum leak the needle valve can provide in a reliable way.
${ }^{29}$ M. Trigo, A. Bruchhausen, A. Fainstein, B. Jusserand, and V. ThierryMieg, Phys. Rev. Lett. 89, 227402 (2002).

${ }^{30}$ G. Rozas, B. Jusserand, A. Fainstein, and A. Lemaître, Chin. J. Phys. 49, 111 (2011).

${ }^{31}$ G. Rozas, M. F. Pascual Winter, B. Jusserand, A. Fainstein, E. Semenova, A. Lemaître, R. Ouillon, and P. Ranson, AIP Conf. Proc. 1199, 169 (2010). 